\title{
Disruption of CTCF at the miR-125b1 locus in gynecological cancers and breast cancer cell lines
}

\author{
Ernesto Soto-Reves*, Fernanda Cisneros-Soberanis, Iliana Alcalá Moreno, Rodrigo Gonzaílez Barrios, \\ David Cantú de León, Clementina Castro, Luis A Herrera \\ From Epigenetics and Chromatin: Interactions and processes \\ Boston, MA, USA. 11-13 March 2013
}

\section{Background}

In cancer cells, transcriptional gene silencing has been associated with genetic and epigenetic defects. The disruption of DNA methylation patterns and covalent histone marks has been associated with cancer development. In non-neoplastic cells the multifunctional CCCTC-binding factor (CTCF) can serve as a barrier against the spread of DNA methylation and histone repressive marks into promoter regions of tumor suppressor genes such as BRCA1, $R b, p 16$ and $p 53$. The absence of CTCF has been related with its epigenetic gene silencing. Until recently, microRNA (miRNA) gene silencing was not well understood. In particular, miR-125b1 has been suggested to be a miRNA with tumor suppressor activity, and it has been shown to be deregulated in various human cancers.

\section{Materials and methods}

As a first approach we performed a functional promoter characterization of a $\mathrm{CpG}$ island proximal to the transcription star site of the miR-125b1 gene. After, demonstrating the promoter activity, we focused in evaluating the DNA methylation of this region in cancer cell lines as well as in normal tissues and gynecological tumor samples. We determine the effect of DNA methylation at the CpG island of $m i R-125 \mathrm{bl}$ on the expression of this gene, we performed a qRT-PCR assay. In addition, we analyzed the association of CTCF and covalent histone modifications at the miR-125b1 locus.

\section{Results}

Our results demonstrated the dissociation of CTCF at the $m i R-125 b 1$ gene promoter in breast cancer cell lines and in gynecological cancers. The disruption of CTCF in breast cancer cells correlated with the incorporation of repressive histone marks such H3K9me3 and H3K27me3 as well as with aberrant DNA methylation patterns. We observed a significant reduction on the expression of $m i R$ $125 b 1$ in cancer cells in comparison with controls, suggesting that DNA methylation and repressive histone marks at the CpG island might reduce miR-125b1 expression. These effects were observed in other gynecological cancers, including ovarian and cervical tumors.

\section{Conclusions}

A reduction of miR-125b1 expression in cancers, correlated with methylation, repressive histone marks and loss of CTCF binding at the promoter region.

\section{Acknowledgements \\ Grants: PAPIIT (IN213311) and CONACYT (83959 and 182997).}

Published: 18 March 2013

doi:10.1186/1756-8935-6-S1-P79

Cite this article as: Soto-Reves et al: Disruption of CTCF at the miR-

$125 b 1$ locus in gynecological cancers and breast cancer cell lines.

Epigenetics \& Chromatin 2013 6(Suppl 1):P79. 